EPiC Series in Engineering
Volume 2, 2018, Pages 1-13
SUMO 2018- Simulating Autonomous
and Intermodal Transport Systems

\title{
Analysis of the traffic behavior of emergency vehicles in a microscopic traffic simulation
}

\author{
Laura Bieker-Walz ${ }^{1}$, Michael Behrisch ${ }^{1}$, and Marek Junghans ${ }^{1}$ \\ German Aerospace Center (DLR) \{laura.bieker, michael.behrisch, marek.junghans\}@dlr.de
}

\begin{abstract}
This paper presents how emergency vehicles can be modeled and simulated in the microscopic traffic simulation SUMO (Simulation of Urban MObility). The special rights of emergency vehicles are implemented in the SUMO framework and can be switched off and on in the simulation with a blue light device. The surrounding traffic reacts accordingly to the emergency vehicle and form an emergency lane. In addition real world data from emergency vehicles are used to evaluate the driving behavior of emergency vehicles and compare it to real world and simulated vehicle characteristics. The evaluation results show that the simulated vehicles pass an intersection generally faster than in real world. For emergency vehicle a time saving of in average one second at a single intersection could be measured for right turning vehicles.
\end{abstract}

\section{Introduction}

Driving an emergency vehicle is a difficult task. On the one hand side, the emergency vehicle needs to reach its destination as quick as possible. On the other hand side, drivers need to take care to avoid any conflicting situations and crashes to reach the destination safely. Unfortunately, emergency vehicles are more likely to be involved in a car accident than other traffic participants. While driving with blue flashing light and siren the likelihood of being involved in a fatal crash is 4 times higher compare to normal vehicles [18].

To evaluate support strategies for emergency vehicles it can help to test and evaluate these strategies in a traffic simulation before implementing them in a real world scenario. At the moment there is only little research published about traffic simulations and models of emergency vehicles. Common microscopic traffic simulation frameworks are modeling the normal traffic behavior of personal vehicles with some limitations and extensions. This means that normally no traffic conflicts like traffic accidents are explicitly modeled. The used models try to add parameters to the model to make it more realistic in comparison to human drivers. This includes reaction times and the imperfection of the human drivers. As a matter of fact, in simulations the drivers often obey all traffic and road restrictions even if human drivers are often speeding or reacting dynamically to unclear traffic situations. Therefore, the modeling of emergency vehicles need also such modifications of the traffic model to allow the vehicles to break common road rules.

E. Wießner, L. Lücken, R. Hilbrich, Y.-P. Flötteröd, J. Erdmann, L. Bieker-Walz and M. Behrisch (eds.), SUMO2018 (EPiC Series in Engineering, vol. 2), pp. 1-13 
An implementation of an emergency vehicle model in CORSIM was presented in [23]. It includes a specific driving behavior algorithm at intersections e.g. the emergency vehicles can violate a red traffic light if other traffic participants are cooperating. The cooperation depends on a random factor if the drivers become aware of the emergency vehicle and how aggressive the drivers are. Furthermore, the emergency vehicles are free to turn in every direction at intersections and are allowed to drive at the opposite side of a road. The opposing driving is realized with a non-existing lane which can only be used by the emergency vehicle.

Another model of emergency vehicles was evaluated and implemented in [21]. The traffic dynamics of vehicles in SUMO were adapted to the traffic behavior of emergency vehicles via a vehicular communication application. The application includes functions to ignore a red traffic light and to force other traffic participants to change the lane to make way for the emergency vehicle.

In addition there are several research results available about the routing and prioritization of emergency vehicles without a proper simulation model of emergency vehicles. Sometimes the reason is the lack of such a simulation model [21] and sometimes the reason is that emergency vehicles have a higher risk being involved in a car crash when they are using their special rights. Therefore, the emergency vehicle support should clear the way so that the emergency vehicle do not have to use their special rights [1].

This paper is structured as follows: first a brief introduction of the used Car-following models is given. Second, the implementation of an emergency vehicle model in SUMO is presented. Third, the simulation scenario and the used traffic data for the evaluation of the vehicle model is stated. Next, the results of the evaluation are stated and discussed. Finally, a conclusion and an outlook about further works is given.

\section{Traffic Simulations}

Traffic simulations are often used to simulate complex traffic situation, to evaluate traffic management strategies, and to predict traffic behavior. This section is based on research which where published in [2] and is extended for this publication. There are already a large scale of traffic simulation frameworks available. For the simulation of the special driving behavior of emergency vehicles it is necessary to model each vehicle individually. Therefore, in this study only microscopic traffic models have been considered.

Many microscopic traffic simulation tools are based on car-following models, which are well studied in research [3]. They focus on the idea that the speed of a vehicle highly depends on the speed of the leading vehicle. The basic idea of the car-following theory is that the change in velocity $v$ of a vehicle $i$ depends on the velocity of the leading vehicle $i+1$ as well as the position difference (gap) and static parameters like the sensitivity or reaction time $\tau$. [10]

$$
\frac{d v_{i}(t)}{d t}=f\left(v_{i+1}(t), x_{i+1}(t)-x_{i}(t), \tau, \ldots\right)
$$

\subsection{Krauss Model}

In the Krauss model each vehicle can have two different motion states:

1. Free motion: no leading vehicle limits the speed of the following vehicle. Its speed is bounded only to its maximum (depending on the speed limit and the drivers desired speed) 
2. Interacting motion: the vehicle has to adapt the speed to the leading vehicle.

The model is without collisions, which means that no vehicle is driving faster than a safe speed $v_{\text {safe }}$. The safe velocity will be computed every time step using the following equation ([9]):

$$
v_{\text {safe }}(t)=v_{l}(t)+\frac{g(t)-v_{l}(t) \tau}{\frac{v}{b(v)}+\tau}
$$

$t:$ time step

$v$ : velocity

$v_{l}(t)$ : velocity of the leading vehicle in $t$

$g(t)$ : gap between vehicle and leading vehicle $i$ in $t$

$\tau$ : reaction time of the driver (usually 1 second)

$b(v)$ : deceleration function

In real life the acceleration of a vehicle depends on its physical ability and other effects (e.g. air resistance). To prevent that vehicles in the simulation are driving faster than possible, the desired speed $v_{\text {des }}$ is calculated. The desired speed of each vehicle $v_{d e s}$ is the minimum speed of the safe speed $v_{\text {safe }}$, the current speed plus the maximum acceleration $a_{\max }$ and the maximum speed $v_{\max }[9]$ :

$$
v_{\text {des }}(t)=\min \left[v_{\text {safe }}(t), v(t)+a, v_{\max }\right]
$$

A value for the imperfection of the driver $\epsilon$ which is multiplied with the acceleration $a$ is subtracted from the desired speed $v_{\text {des }}$ [9]. For the velocity a random value is chosen (with the function rand) between: imperfect desired speed $v_{\text {des }}(t)-\epsilon a$ and the desired speed $v_{\text {des }}(t)$ :

$$
v(t)=\max \left[0, \operatorname{rand}\left[v_{\text {des }}(t)-\epsilon a, v_{\text {des }}(t)\right]\right]
$$

\subsection{Intelligent Driver Model - IDM}

The Intelligent Driver Model (IDM) is based on the Optimal Velocity Model (OVM) [17]:

$$
\dot{v}=\frac{\left(v_{o p t}(g)-v\right)}{\tau}
$$

where: $\dot{v}$ : acceration function

$g:$ current gap to the leading vehicle

$v_{\text {opt }(g)}:$ optimal velocity depends on gap $s$

$\tau$ : Time to adapt to the new speed

The speed of the leading vehicle is not taken into consideration by the OVM which reacts only to the distance of the leading vehicle. An extension of the OVM was modeled because it is very sensitive to accidents. The new model was the IDM which has the following equation for the acceleration $[15,16]$ :

$$
\dot{v}=a\left[1-\left(\frac{v}{v_{\text {des }}}\right)^{\delta}-\left(\frac{g^{*}(v, \Delta v)}{g}\right)^{2}\right]
$$

where: $v:$ current velocity

$v_{\text {des }}:$ desired velocity 


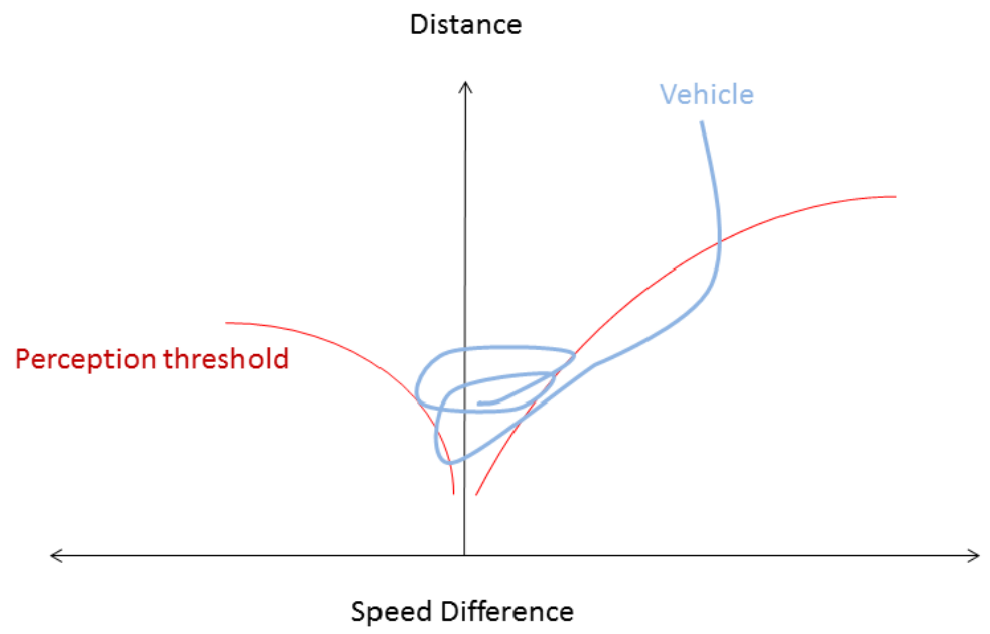

Figure 1: Perception thresholds in the Wiedemann model with an example vehicle [22]

$g^{*}:$ desired gap

$\delta$ : acceration exponent, usually set to 4

The desired gap $s^{*}$ is calculated as follows:

$$
g^{*}(v, \Delta v)=s_{0}+\max \left[0,\left(v T+\frac{v \Delta v}{2 \sqrt{a b}}\right)\right]
$$

Every vehicle can have other values for the parameter of the model:

$T$ : the time headway (between $0.8-2$ seconds)

$g_{0}:$ is the minimum gap (default: 2 meters)

$a$ : acceleration (between $1-2 \mathrm{~m} / \mathrm{s}^{2}$ )

$b$ : deceleration (between $1-2 \mathrm{~m} / \mathrm{s}^{2}$ )

\subsection{Wiedemann}

The Wiedemann Model is used in the commercial and well-known traffic simulation Vissim [12]. The basic approach of the model is a psycho-physical spacing model. If a faster vehicle is approaching a slower leading vehicle, it will start to decelerate until it reaches its individual threshold. The threshold is a function of speed difference and spacing. While human drivers are not very good in perceiving small speed differences and in keeping their speed very accurate, the model uses two different threshold for acceleration and deceleration [5]. A schematic figure of the thresholds and one example vehicle are depicted in Figure 1

\section{$3 \quad$ Emergency vehicle modeling}

One focus of this paper is to implement the application functions of Weinert et. al. [21] and other special rights of emergency vehicles into the core simulation of SUMO. The described 
model are implemented in SUMO and can be used with a blue light device. A blue light device can be given explicitly a vehicle or with a probability. With the blue light device the simulation knows that the vehicle is allowed to use special rights. The device can be switched off again if the vehicle is not on duty and the emergency vehicle should react like a normal vehicle.

In addition, a graphical shape for emergency vehicle has been implemented. With the vTypeattribute "guiShape" an emergency vehicle shape can be set for the vehicle. When setting the attribute to "emergency" a white ambulance with the international first aid sign is displayed in the graphical user interface of SUMO in the "real world" modus. Furthermore, a police car can be chosen with the attribute "police" and a fire brigade with guiShape= "firebrigade". For a better overview the parameter and the description for the emergency vehicle configuration are listed in Table 1.

\begin{tabular}{llll} 
Configuration File & Attribute & Value & Description \\
\hline vType & guShape & $\begin{array}{l}\text { emergency } \\
\text { police } \\
\text { firebrigade }\end{array}$ & $\begin{array}{l}\text { Emergency vehicle visualization } \\
\text { Police car visualization } \\
\text { Visualization of a fire brigade }\end{array}$ \\
\hline vType & vClass & emergency & $\begin{array}{l}\text { Blue flashing light and } \\
\text { special rights for driving on every lane }\end{array}$ \\
\hline vType & speedFactor & $>1.0$ & Allows to drive faster than speed limit \\
\hline SUMO config & $\begin{array}{l}\text { device. } \\
\text { bluelight.explicit }\end{array}$ & vehicleID & $\begin{array}{l}\text { Allows to use emergency lanes and } \\
\text { violate red traffic lights etc. }\end{array}$ \\
\hline
\end{tabular}

Table 1: Summary of the emergency vehicle configuration

There is also a vehicle Type implemented for emergency vehicles which is set by vClass="emergency" in the vType definition. The vehicle class also triggers the blue flashing light and the restrictions for special lanes.

In the following sub-sections different aspects of the traffic behavior of emergency vehicles are described and how they have been implemented in SUMO:

\section{$3.1 \quad$ Speeding}

While private vehicles have to keep the local speed limits, emergency vehicle are allowed to drive faster as long they are not jeopardize themselves or other traffic participants. This means that even if emergency vehicles could drive with $200 \mathrm{~km} / \mathrm{h}$ in the city during the peak hour, they are not allowed to because the traffic conditions are not safe to do it. Because of this it makes sense that emergency vehicles should not be modeled in a way that they are always driving with maximum possible speed. A more realistic way is to model a speed distribution which allows exceeding the speed limit.

The exceeding of the speed limit is supported in SUMO via an attribute called speedFactor in the vType definition. With a value of 1.5 the vehicles are allowed to drive with $150 \%$ of the allowed road speed limit.

\subsection{Using an emergency lane}

When an emergency vehicle wants to use a virtual middle lane, other traffic participants have to build an emergency lane before. For this a sub-lane model of the traffic network has to be used. Otherwise, all traffic participants are always driving in the middle of the lane. For the 


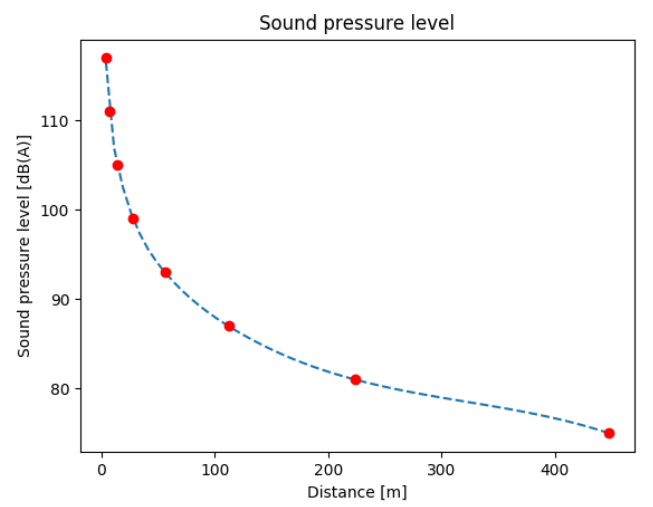

Figure 2: Sound pressure level in relation to the difference of the siren from the emergency vehicle

cooperative traffic behavior of other vehicles a reaction time when they react to an approaching emergency vehicle has to be estimated. Therefore, the evaluation results of the study of Golder [6] were used. Golder performed tests when the siren of an emergency vehicle could be perceived. In Figure 2 the sound pressure level of the siren is depicted. Golder also states that the sound pressure level at the location of the vehicle has to be higher than $100 \mathrm{~dB}(\mathrm{~A})$ if the radio inside the vehicle is switched on or people are talking with each other (this takes another $70 \mathrm{~dB}(\mathrm{~A})$ ) and the vehicle body are normally so good isolated that they lower the sound pressure level again by $30 \mathrm{~dB}(\mathrm{~A})$. Thus, in average the vehicles should realize an approaching emergency vehicle within distance of 25 meters.

In the simulation all vehicles are reacting to the emergency vehicle and are forming an emergency lane by changing the sub-lanes. If the vehicle is on the left most lane it changes the sub-lanes to left and for all others to right. While the emergency vehicle is approaching all other vehicles are not allowed to use the emergency lane. After the emergency vehicle passed the vehicles they continue their normal driving. An example scenario of an emergency lane is illustrated in Figure 3.

\subsection{Violating red traffic lights}

The normal behavior of a vehicle in SUMO is reducing speed at an intersection and following the right-of-the way rules or break at a red traffic light. With speed mode command in TraCI [20] (the interface for online interaction in SUMO) these safety checks and rules can be disregards. This also implies for an emergency vehicle with blue light device which can by default pass red traffic lights.

\subsection{Overtaking}

In SUMO emergency vehicles are always permitted to overtake on the right when the vehicle has the vClass attribute "'emergency"'. In addition it is possible to allow overtaking at the opposing lane. But the combination of this with the sublane model is currently not working and should be implemented in further work. 


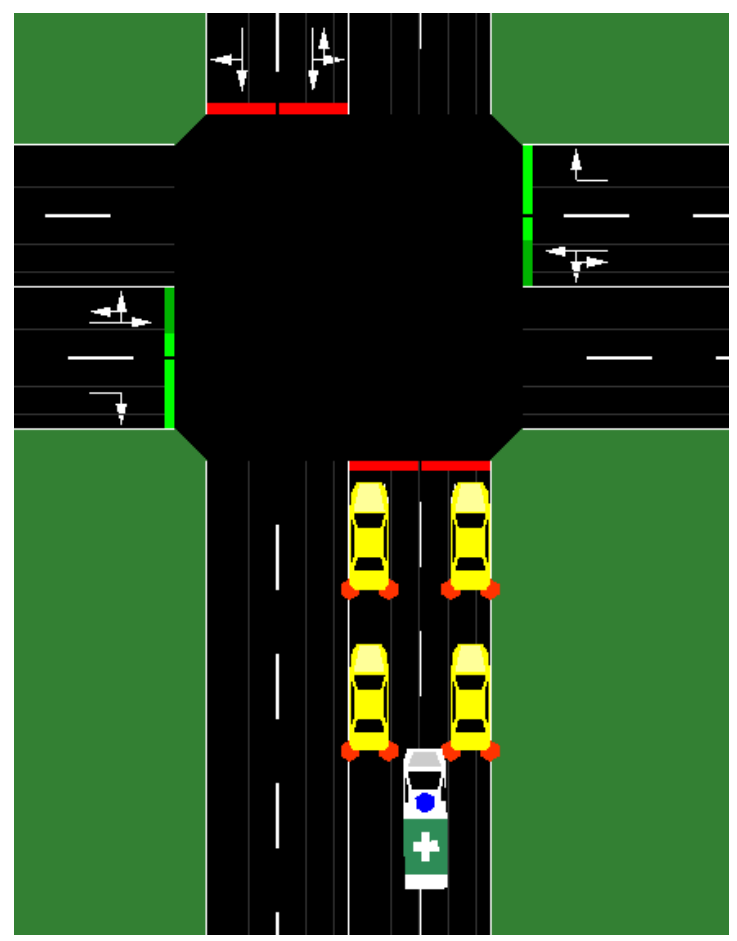

Figure 3: Forming an emergency lane for an emergency vehicle in SUMO

\section{Driving Behavior of Emergency Vehicles}

To validate the emergency vehicle model an evaluation of the normal private traffic compare to real emergency vehicle drivers were done. Therefor a simulation scenario of a real world intersection was set up and simulated. The results of the simulation were compared to the real world trajectories from private cars and to the trajectories from real emergency vehicles. In the following the simulation scenario and the used traffic data are described.

\subsection{Simulation scenario}

For this study SUMO 0.32 was used [8] to simulate the intersection in Brunswick, see Figure 4a.

The default car-following model of SUMO is the Krauss model [10, 11]. For comparison not only the default Krauss model was used in addition all simulations were also run with the IDM and Wiedemann model. The vehicle trajectories were exported as floating car data (FCD) in XML format.

\subsection{Real World Traffic Data}

For the real world traffic a highly frequented junction ( $>20,000$ traffic participants per day) in Brunswick, Germany, has been selected. The trajectory data (space-time-curves) of human drivers have been recorded at this controlled intersection which includes two main roads. For the evaluation one hour from 23. January 2017 of this research intersection were used. The research intersection was set up for the test field AIM (Application Platform for Intelligent 


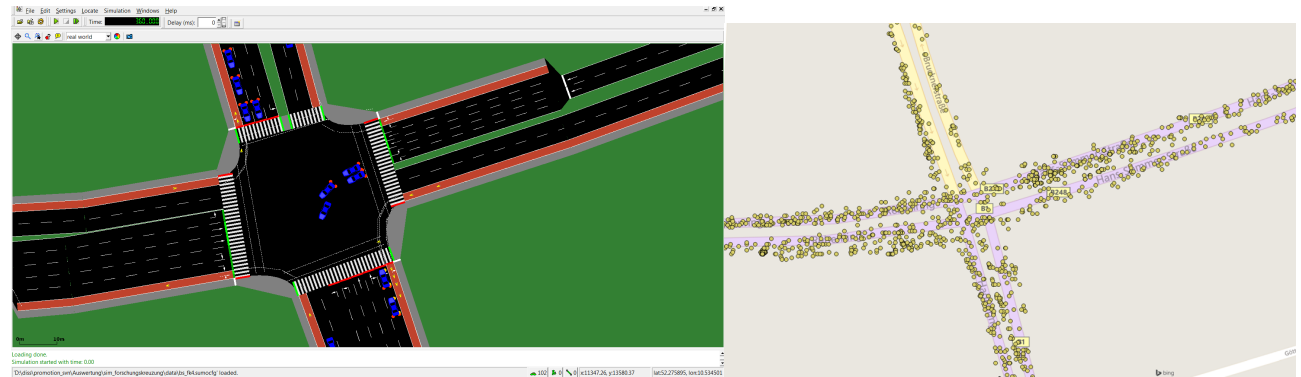

(a) SUMO Simulation of the analyzed intersection in(b) Emergency vehicle data at the analyzed Brunswick [2] intersection in QGIS [4]

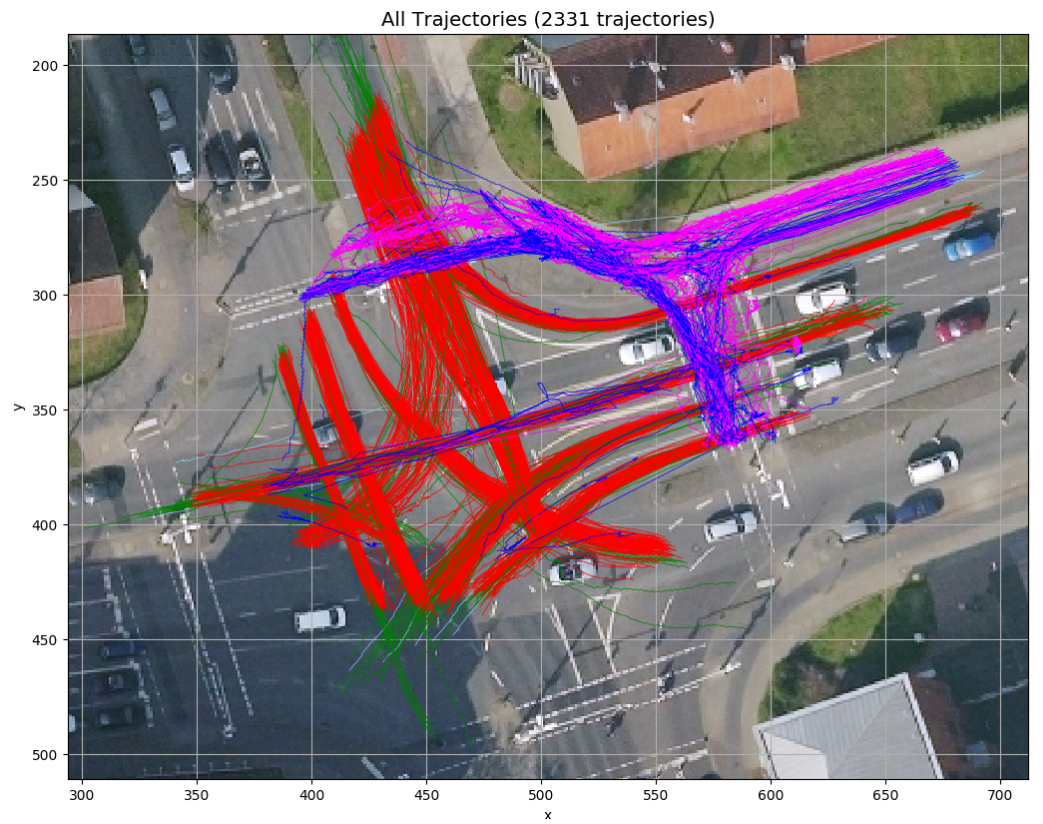

Figure 4: Real world picture of the intersection in Brunswick (Lines are representing detected trajectories red: vehicles, blue: bikes, pink: pedestrians) [2]

Mobility) provides a research instrument to detect and asses the traffic behavior at a complex controlled and highly frequented intersection [7].

For the traffic monitoring several mono-cameras and multirange radar sensors are installed to detect and track traffic participants during their crossing of the intersection. Furthermore, the tracked trajectories include information about the different vehicle types (cars, motorcycles, trucks/vans, bicycles and pedestrians), their size, kinematic states (position, speed, acceleration) and headings. For more details see [7] and [14]. For the evaluation in this research the vehicle position and speeds extracted from the video cameras were used.

In addition to the camera data of all traffic participants at the single intersection, also trajectory data of emergency vehicle of the city of Brunswick were collected and analyzed for this research. The evaluation period includes the time from October 2013 until February 2018. The trajectories of the emergency vehicles at this intersection includes over 7,000 emergency 
vehicle routes. The Position data of 24 emergency vehicles are tracked every 5 seconds and also sensor data e.g. state of the siren and blue light are also available. An example visualization of the trajectory data can be seen in Figure $4 \mathrm{~b}$.

\section{Results}

For the evaluation in this research the trajectory data of private vehicles from real world cameras are compared with the emergency vehicle data and the simulated vehicles in SUMO. The simulation with SUMO was performed with three different car-following models: SUMOs default car-following model Krauss, IDM and Wiedemann. A box whisker plot of the average speeds over the intersection can be seen in Figure 5.

As expected the average speed for right turning emergency vehicles is slightly higher (around $7 \mathrm{~km} / \mathrm{h}$ ) than for private vehicles. The reason for this is probably that the speed of private vehicles while right turning are normally reduced by the interaction with other traffic participants especially pedestrians which are also crossing the intersection. In contrast to this, pedestrians are normally hearing an approaching emergency vehicle and are waiting at the pathway until the emergency vehicle passed the intersection.

Interestingly, all simulation models are over estimating the average speed the vehicle could pass an intersection. One reason for this is that there might be not enough pedestrians simulated to block the vehicles. Additionally, human drivers might drive more carefully to have more time to react to unforeseen events. The traffic simulation has full knowledge of all traffic participants and knows that no pedestrian is close, but in real world drivers have to stop or reduce their speed first to make sure that there is no other traffic participant in blind spot. Another factor is: vehicles in SUMO are not reducing their speed while driving curves in contrast to human drivers would do. This aspect can also be seen in evaluation results in Figure 5. The speed differences between human drivers and the simulation is much higher for right turning vehicles.

The passing times the vehicles needed to cross the intersection are stated in Figure 6. As expected, the results are similar to the evaluation of average speeds at the intersection. It can be seen that emergency vehicles are saving around one second while turning right at an intersection. But if the emergency vehicle just follow the main road the spreading of the data is much larger than for private vehicles. In addition, there are many outlines: some emergency vehicle needed over 60 seconds to cross the intersection while normal vehicles normally need less than 10 seconds. There could be different reasons for this:

- for example the emergency vehicles are blocked by other traffic participants. Only the trajectories of the vehicle which passed the stopping line have been evaluated here, because of the angle of the traffic camera. Therefore, it can be assumed that all vehicles detected by the traffic camera a driving while the traffic light is green. For the emergency vehicle this must not be necessary the case. Because of their special rights it could be that the emergency vehicle violates the red traffic light and has to stop because of other traffic participants block the intersection.

- Another reason could be that an accident at this intersection happened or the intersection was the destination of the emergency vehicle. 


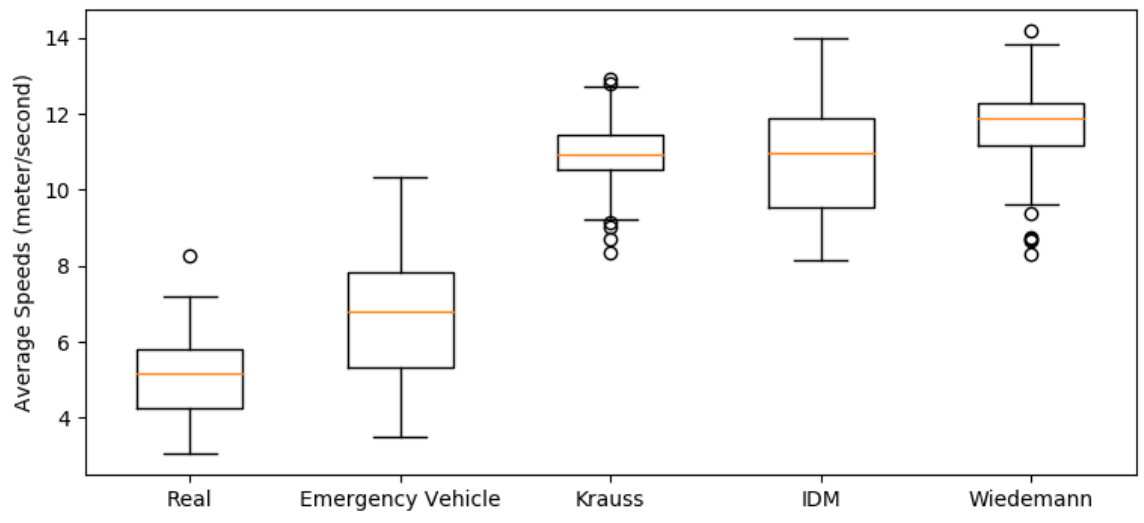

(a) Right turning

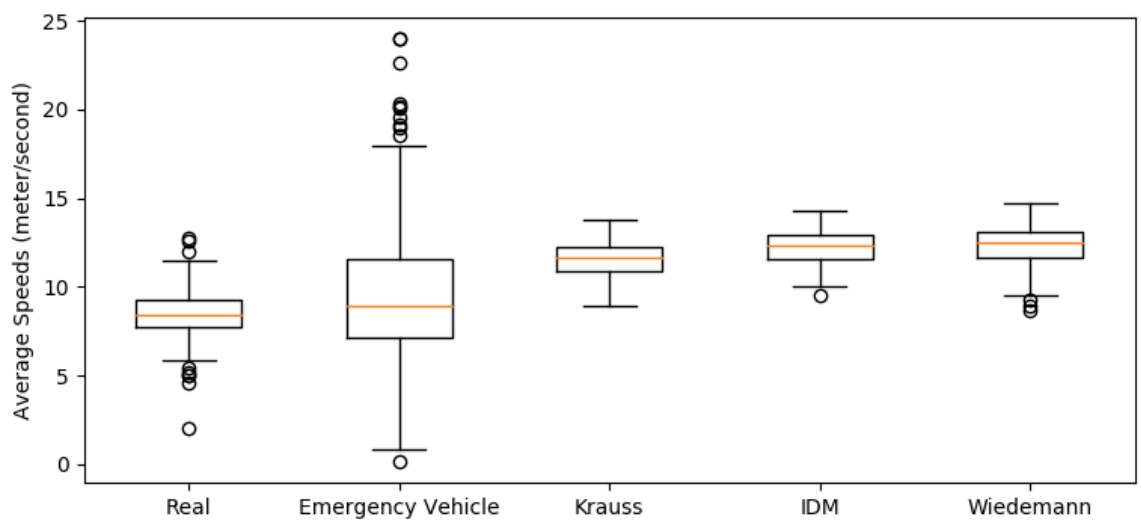

(b) Straight driving

Figure 5: Average Speed of the vehicles while crossing the intersection

\section{Conclusion and Future Work}

In this research a model for emergency vehicles have been introduced. But the model still has some limitations and further research is necessary. As the evaluation results indicate, the simulated travel times over the intersection are in general faster than in real world. These results should be used to modify the current simulation models in SUMO to make them more realistic.

One general limitation of simulations is that they model the average traffic behavior and not necessarily the real human behavior of one individual traffic participant. For example modeling of the emergency lane formation presume that the surrounding traffic reacts accordingly to the approaching emergency vehicle. Lately, there have been many reports in the news about people who do not form an emergency lane properly or used the emergency lane for their own advantage $[13,19]$. This misbehavior is not modeled here. 


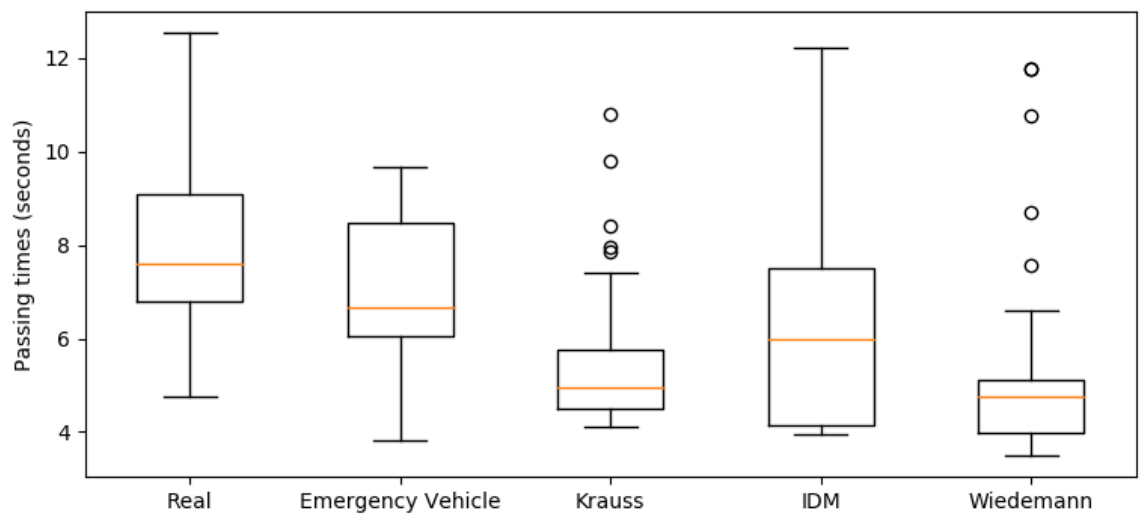

(a) Right turning

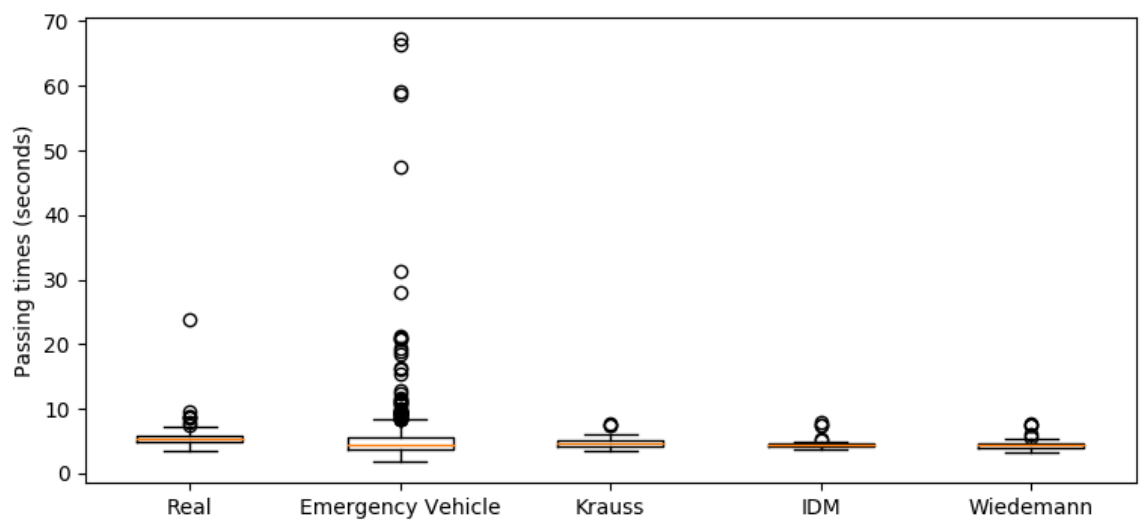

(b) Straight driving

Figure 6: Passing times of the vehicles while crossing the intersection

The presented paper gives an overview of an emergency vehicle model implemented in the simulation framework SUMO. The implemented model allows user to simulate their own traffic management strategies with emergency vehicles. The model still has some limitations and will be extended in further work. For example a realistic default value for speeding should be set automatically to the emergency vehicle model. In addition for the emergency vehicles the sublane model and the overtaking at the opposing lane function should be combined to allow both in the same simulation scenario. Furthermore, a proper calibration of the emergency vehicle model is planed.

The presented emergency vehicle model gives researchers a starting point to simulate and work on emergency vehicle models and the support for emergency vehicle drivers. 


\section{References}

[1] Laura Bieker-Walz. Cooperative traffic management for emergency vehicles in the city of bologna. In SUMO 2017 - Towards Simulation for Autonomous Mobility, volume 31 of Berichte aus dem DLR-Institut für Verkehrssystemtechnik, pages 135-141, Mai 2017.

[2] Laura Bieker-Walz, Michael Behrisch, Marek Junghans, and Kay Gimm. Evaluation of carfollowing-models at controlled intersections. In European Simulation and Modelling Conference, volume 31, pages 247-251, Oktober 2017.

[3] Mark Brackstone and Mike McDonald. Car-following: a historical review. Transportation Research Part F: Traffic Psychology and Behaviour, 2(4):181 - 196, 1999.

[4] QGIS Contributors. Qgis user guide, 2018. last retrieved 8 March 2018.

[5] Martin Fellendorf. Vissim: A microscopic simulation tool to evaluate actuated signal control including bus priority. In 64th Institute of Transportation Engineers Annual Meeting, pages 1-9. Springer, 1994.

[6] Uwe Golder. Unfälle mit fahrzeugen mit sonderrechten. VerkehrsRechtsReport, (9), 2008.

[7] Sascha Knake-Langhorst and Kay Gimm. Aim research intersection: Instrument for traffic detection and behavior assessment for a complex urban intersection. Journal of large-scale research facilities, 2(A65), 2016.

[8] Daniel Krajzewicz, Jakob Erdmann, Michael Behrisch, and Laura Bieker. Recent development and applications of SUMO - Simulation of Urban MObility. International Journal On Advances in Systems and Measurements, 5(3\&4):128-138, December 2012.

[9] Daniel Krajzewicz, Georg Hertkorn, C. Rössel, and Peter Wagner. Sumo (simulation of urban mobility) - an open-source traffic simulation. In A. Al-Akaidi, editor, 4th Middle East Symposium on Simulation and Modelling, pages 183-187, 2002. LIDO-Berichtsjahr=2004,.

[10] Stefan Krauss. Microscopic Modeling of Traffic Flow: Investigation of Collision Free Vehicle Dynamics. PhD thesis, Universität zu Köln, 1998.

[11] Stefan Krauss, Peter Wagner, and Christian Gawron. Metastable states in a microscopic model of traffic flow. Phys. Rev. E, 55:5597-5602, May 1997.

[12] Sandeep Menneni, Carlos Sun, and Peter Vortisch. Integrated microscopic and macroscopic calibration for psychophysical car-following models. In Transportation Research Board 88th Annual Meeting, number 09-2773, 2009.

[13] Stuttgarter Nachrichten. Motorradfahrer fáhrt durch rettungsgasse. accessed 21. March 2018.

[14] Lars Schnieder and Karsten Lemmer. Anwendungsplattform intelligente mobilität-eine plattform für die verkehrswissenschaftliche forschung und die entwicklung intelligenter mobilitätsdienste. Internationales Verkehrswesen, 64(4):62-63, 2012.

[15] Martin Treiber. Longitudinal traffic model: The idm, 2017.

[16] Martin Treiber, Ansgar Hennecke, and Dirk Helbing. Congested traffic states in empirical observations and microscopic simulations. pre, 62:1805-1824, aug 2000.

[17] Martin Treiber and Arne Kesting. Verkehrsdynamik und -simulation: Daten, Modelle und Anwendungen der Verkehrsflussdynamik. Springer-Lehrbuch. Springer Berlin Heidelberg, 2010.

[18] Manfred Unterkofler and Reinhard Schmiedel. Verbesserung der sicherheit bei sondersignaleinsätzen. bergisch-gladbach, info 34/95. Wissenschaftliche Informationen der Bundesanstalt für Straßenwesen, 34/95, 1995.

[19] WDR. Feuerwehr musste rettungsgasse auf a2 selbst organisieren. accessed 21. March 2018.

[20] Axel Wegener, Michał Piórkowski, Maxim Raya, Horst Hellbrück, Stefan Fischer, and Jean-Pierre Hubaux. Traci: an interface for coupling road traffic and network simulators. In Proceedings of the 11th communications and networking simulation symposium, pages 155-163. ACM, 2008.

[21] Florian Weinert and Michael Düring. Development and assessment of cooperative v2x applications for emergency vehicles in an urban environment enabled by behavioral models. In Michael Behrisch 
and Melanie Weber, editors, Modeling Mobility with Open Data, pages 125-153, Cham, 2015. Springer International Publishing.

[22] Rainer Wiedemann. Simulation des Straßenverkehrsflusses", Schriftenreihe des Instituts für Verkehrswesen der Universitä Karlsruhe, Heft 8. PhD thesis, 1974.

[23] Li Zhang, Jizhan Gou, Ke Liu, Gene McHale, Raj Ghaman, and Ling Li. Simulation modeling and application with emergency vehicle presence in corsim. In Vehicular Technology Conference Fall (VTC 2009-Fall), 2009 IEEE 70th, pages 1-7. IEEE, 2009. 